\title{
Assessing cardiovascular links to depression and anxiety in Australian professional drivers
}

\author{
Taryn Chalmers ${ }^{1}$, Sara Lal ${ }^{1, *}$ \\ ${ }^{1}$ Neuroscience Research Unit, School of Life Sciences, University of Technology Sydney, 2007 Ultimo, New South Wales, Australia \\ *Correspondence: Sara.Lal@uts.edu.au (Sara Lal)
}

DOI:10.31083/j.jin2101043

This is an open access article under the CC BY 4.0 license (https://creativecommons.org/licenses/by/4.0/).

Submitted: 10 April 2021 Revised: 26 April 2021 Accepted: 24 May 2021 Published: 28 January 2022

Train and truck drivers experience a myriad of unique occupational factors, which have been postulated to contribute to a high incidence of health conditions such as depression anxiety and cardiovascular disease amongst this population. The present study aimed to identify associations between heart rate variability and negative mood states such as depression and anxiety in a cohort of Australian truck and train drivers. 120 professional drivers (60 truck drivers, 60 train drivers) were recruited from the local community. Participants complete a battery of psychometric questionnaires to assess levels of negative mood states such as depression and anxiety. Participants then completed a baseline (resting) and active (driving) task while concurrent electrocardiography data was collected to obtain heart rate variability parameters. Anxiety and depression were found to be associated with increases in low frequency heart rate variability and sympathovagal balance, and a reduction in total power. The present study identified associations between negative mood states and heart rate variability parameters that are unique to this cohort.

\section{Keywords}

Heart rate variability; Professional driver; Truck driver; Train driver; Depression; Anxiety

\section{Introduction}

Australia's rail and road freight systems are primary means for the movement of freight and people between cities, states and territories. These industries employees over 1 million individuals each year, and experience annual industry growth of approximately 4\% [1]. Given that Australia's person per square kilometer of land ratio is amongst the lowest in the world [2], a reliance on road and rail freight has persevered despite advancements in commuter and transport technology.

Train and truck drivers experience a myriad of unique occupational workplace factors, such as monotonous driving conditions, long hours spent sitting, the necessity of strict mental alertness, workplace social isolation and the potential for "person under vehicle" events. These conditions have been postulated to contribute to a high incidence of health conditions such as depression [3-9], anxiety [10-13] and cardiovascular disease (CVD) [14-16] amongst this population.

Although often occurring independently of one another, the link between depression and cardiovascular risk is well established. The world health organisation recently identified depression as in independent risk factor for heart disease [17]. Individuals with depression are more likely to suffer from CVD and are more likely to die from CVD related diseases than the general population [18]. The mechanisms by which depression and CVD are linked, however, remain somewhat elusive. Various pathophysiological mechanisms have been suggested, including increased inflammatory responses; hypercoagulability as a result of deleterious adaptions of the clotting cascade; upregulation of oxidative stress responses; hyperactivity of the hypothalamic-pituitaryadrenal axis; downregulation of systemic endothelial progenitor cells; decreased heart rate variability; and genetic diatheses [19-22]. Recent studies have utilised heart rate variability (HRV), the analysis of minute fluctuations in successive heart beats, as a means of quantifying cardiovascular autonomic control. In particular, HRV can be used to measure parasympathetic nervous system activity and has been utilised as an indirect measure of cardiovascular function. Previous studies assessing the associations between depressive symptomology and HRV have yielded varied results, however limitations in sample size, psychometric assessment and potential reporting bias may have obscured results.

Given the high rates of both depression and CVD within the population of Australian truck and train drivers, this study aimed to identify associations between heart rate variability and negative mood states such as depression and anxiety in a group of Australian truck and train drivers. Quantitative assessment of the association between depression and cardiovascular function (as measured by HRV) in the Australian professional driving community may provide the foundation for a dual mitigation approach within the workplace to manage both conditions within this community.

\section{Methods \\ 2.1 Participants}

A total of 60 truck drivers (mean age $36.5 \pm 9.67, \mathrm{n}=45$ males, $\mathrm{n}=4$ females) and 60 train drivers (mean age 39.16 $\pm 10.51, \mathrm{n}=53$ males, $\mathrm{n}=5$ females) were recruited for assessment in this cross-sectional study. Participants were recruited through local advertisement via a poster, recruitment 
through contacts established independently to this research, online forums and with the aid and endorsement of Australia Post Transport Division, Sydney Trains and the Australian Trucking Association. Participants were required to be employed as a truck driver, regularly driving a truck with a gross vehicle mass of over 4.5 tonne, or a currently employed train driver.

\subsection{Procedure}

Participants were tested between 9.30 AM and 3 PM in order to negate the variations in heart rate between 8-9 AM and 4-8 PM [23]. The inclusion criteria were as follows; current employment as a full-time truck or train driver, and fluent English literacy. The exclusion criteria, as determined by part one of the Lifestyle appraisal questionnaire [24], were as follows; those currently taking any prescription or nonprescription drugs (excluding caffeine and nicotine) or suffering from any chronic disease or illness, were excluded from the study. Participants were required to abstain from food for 2 hours, nicotine and caffeine for 4 hours, and alcohol for 12 hours before the study; to avoid influencing the physiological measures. The study was conducted in a controlled laboratory environment, with auditory and visual interference being reduced as much as viably possible. Light sources were controlled, with laboratory blinds being drawn to reduce the impact of external light sources influencing physiological measurements. The study was comprehensively detailed to the subject upon arrival, with the opportunity for questions being presented. Upon confirmation of written consent, the study was commenced.

\subsection{Questionnaires}

The questionnaire package included the in-house designed Professional Driver Package, Lifestyle Appraisal Questionnaire [24], the Becks Depression Inventory [25], and the Profile of Mood States Questionnaire [26]. The Professional Driver Package provides demographical information regarding licensing, employment length, employment status, nutrition, accident or near-miss history and working conditions. The Lifestyle Appraisal Questionnaire (LAQ), a validated and clinically reliable questionnaire, was used to record demographic, lifestyle and psychological stress information from the participant. The LAQ consists of two parts, with Part I consisting of 22 questions, with the highest obtainable score being 73 . This information included family history of disease, smoking status, alcohol intake, exercise and diet regime, etc. The higher the score obtained from Part I of the LAQ, the greater the risk of developing a chronic illness later in life. Part II of the LAQ consists of 27 items and assessed an individual's "cognitive appraisal of pressure and demands" [24]. The Beck's Depression Inventory Scale (BDI-II) [25] is a 21-item self-reported tool used to assess the severity of depression in adults and adolescents (aged $>13$ ). Scores range from 0-63, with 0-13 indicating minimal depression, 14-19 indicating mild depression, 20-28 indicating moderate depression and 29-63 indi- cating severe depression [27]. The coefficient alpha of the BDI-II was found to be 0.93 , indicating high internal consistency. The test-retest correlation of the BDI-II was found to be 0.93 , which was significant $(p \leq 0.001)$. As such, the BDI-II is a suitable psychometric tool for the assessment of depressive symptoms within this study. The Profile of Mood States questionnaire (POMS) [26] is composed of 65 items describing six mood subscales: tension-anxiety, depressiondejection, anger-hostility, vigor-activity, fatigue-inertia, and confusion-bewilderment. An overall measure of total mood disturbance is calculated for all six subscales by combining the scores obtained on the tension-anxiety, depression-dejection, anger-hostility, fatigue-inertia, and confusion-bewilderment scales minus the score on the vigor-activity scale. The depression-dejection subscale is strongly predictive of the Beck Depression Inventory-II (BDI-II) [27], which is often used in clinical practice to diagnose depression. The depression-dejection subscale of the POMS is consequently considered a useful short alternative to the BDI-II, since it also investigates other components of mood such as anxiety and aggression [28].

\subsection{Heart rate variability}

Standardised attachment of a three-lead electrocardiogram was performed, with the active electrodes being positioned at the intercostal space between the fourth and fifth ribs, two centimetres laterally from each side of the sternum and the reference electrode being secured underneath the shoulder. Following this, the participant was asked to rest for five minutes, after which, the participant was seated in the driving simulator and after participating in the race format for ten minutes to familiarise themselves with program, were asked to complete the 'active' driving situation, in which they were engaged in a race meeting (Fuji Speedway) with other automatically generated cars whilst a concurrent twenty-minute ECG recording was obtained. The driver was then asked to rest for ten minutes. Following this, a baseline (control) recording was obtained, which involved the participant engaging in a twenty-minute driving session, again on the Fuji Speedway, with the absence of other automobiles on the road whilst a concurrent twenty-minute ECG recording was taken. The driving simulator was employed in order to elicit a physiological stress response, and it should be noted that subjects were familiarised with the program prior to ECG recordings being obtained. Heart rate variability (HRV) data was then obtained from the R-R intervals of the ECG recordings using a non-parametric algorithm (Fast Fourier Transform) and used as a quantitative measurement of the sympathetic (Low Frequency HRV) and parasympathetic nervous systems (High Frequency HRV). HRV reactivity was obtained by calculating the increase from baseline to active states in the various HRV parameters.

\subsection{Heart rate variability analysis}

Initially, using the QRS detector, the pre-processing step of HRV analysis included band pass filtering to decrease 
power line noise, baseline wander, muscle noise and any other interference components. The pass band at approximately $5-30 \mathrm{~Hz}$ is sufficient to cover most of the frequency content of QRS complex [29]. After this pre-processing had occurred, a set of decision rules were applied to define if a QRS complex had occurred. The decision rules included the average heartbeat period as well as the amplitude threshold, which were amended adaptively as the detection process continued. The fiducial point was selected to be the R-Wave, and the time at which the R-Wave occurs was logged. Post $\mathrm{R}$-Waves identification, and time of R-Wave occurrence was determined, the HRV time series was derived. The R- $\mathrm{R}$ intervals were determined as the variances between successive R-Wave occurrence time periods. A power spectrum density (PSD) estimate was then used to calculate the R-R interval series. The PSD estimation is performed using the Fast Fourier Transform based Welch's periodogram method (Hann window was used). In the Welch's periodogram method, the HRV sample is separated into overlapping segments (50\% overlap). The spectrum was then acquired by calculating the average spectra of these segments. This method reduces the amount of variance of the FFT spectrum. The frequency bands derived for short-term HRV recordings were low frequency (LF, 0.04-0.15 Hz) and high frequency (HF, 0.15-0.4 $\mathrm{Hz}$ ). The absolute power values for each frequency band were derived through integration of the spectrum over the band limits.

\subsection{Statistical analysis}

Power analysis was applied to determine the minimum sample size required to produce statistically reliable data. The minimum sample size required for this research, based on moderate-large effect, was 30 [30]. Statistical power increases with increasing sample size $[29,31]$. As such, with a total sample size of 120 , sufficient power for statistical analysis was obtained. The statistical analyses was conducted using the software program SPSS Version 22.0 (IBM Corp, Armonk, NY, USA) and comprised of dependent sample $t$-tests, Pearson's correlations and regression analyses. Associations of significance are presented at a $p$ value of $<0.05$.

\section{Results \\ 3.1 Demographics}

The average age recorded for the professional driver sample was $37.0 \pm 9.5$ years, which was younger than the national average age of employees (46 years). The mean BMI was reported as 28.6 which is within the overweight category (2530) [32]. The sample was $93.3 \%$ male, which reflects the male dominance of this industry. Average years employed as a professional driver was $9.3 \pm 7.4$, and the average hours driving a vehicle as a worker each week was $33.8 \pm 9.7$. Drivers reported $0.9 \pm 1.5$ accidents or near misses in the previous 12 months, and $4.7 \pm 7.3$ accidents or near misses in their career (Table 1).

\subsection{Psychometric tests}

The mean test scores of each of the six respective mood subscales (tension-anxiety, anger-aggression, fatigue-inertia, depression-dejection, confusion-bewilderment and vigoractivity) of the POMS questionnaire, a total mood disturbance scores, along with the normative values [33] are listed in Table 2 (Ref. [33]). It should be noted that through single sample $t$-tests, it was ascertained that scores were significantly higher for tension-anxiety $(p=0.003)$ angeraggression $(p=0.03)$, fatigue-inertia $(p \leq 0.001)$, depressiondejection $(p=0.004)$ and total mood disturbance $(p \leq 0.001)$, and significantly lower for vigor-activity $(\leq 0.001)$ than the advised normative values [33].

The mean BDI-II test score for the professional driving sample $(\mathrm{n}=120)$ are presented in Table 3 (Ref. [25]), along with the cut-off for mild to moderate depression [25].

There was no significant differences in BDI scores between the current sample of professional drivers $(n=120)$ and the advised normative cut-off [25].

\subsection{Baseline task}

The mean HRV (LF, HF, TP and LF:HF) for the professional driving sample $(\mathrm{n}=120)$ during the baseline phase is displayed in Table 4.

Partial correlations, when controlling for gender, smoking status, age and BMI, were undertaken to assess associations between baseline HRV parameters, profile of mood states scores and Beck's Depression Inventory scores. Significant findings are displayed in Table 5.

Seven statistically significant correlations between depression scores and HRV parameters were identified during the baseline task in truck drivers (Table 5). Tension-anxiety and anger-aggression (as measured by the POMS) were found to be correlated with LF, HF and Ration, and TP respectively. The BDI was found to be correlated with LF and TP.

\subsection{Active phase}

The mean HRV (LF, HF, TP and LF:HF) for the professional driving sample $(\mathrm{n}=120)$ during the active phase is displayed in Table 6.

Partial correlations, when controlling for gender, smoking status, age and BMI, were undertaken to assess associations between active HRV parameters, profile of mood states scores and Beck's Depression Inventory scores. Significant findings are displayed in Table 7.

A number of statistically significant correlations between depression scores and HRV parameters were identified during the active task. Confusion bewlderment was correlated with LF and HF. Total mood disturbance was correlated with LF and ratio. BDI was correlated with LF and HF.

\section{Discussion}

Mental Health and links to cardiovascular parameters in Australian professional drivers have rarely been studied, which is of concern given the reportedly high levels of negative health states reported within this occupational group 
Table 1. Demographic data of the professional driving sample $(n=120)$.

\begin{tabular}{lccc}
\hline & Mean \pm SD or \% & Median & Interquartile range \\
\hline & $37.0 \pm 9.5$ & & \\
Gender (Males) & 93.34 & & \\
BMI & $28.6 \pm 4.3$ & & \\
Years employed & $9.3 \pm 7.4$ & & \\
Hours driving & $33.8 \pm 9.7$ & & 1 \\
Accidents/near misses in previous year & $0.9 \pm 1.5$ & 0 & 4 \\
Accidents/near misses per career & $4.7 \pm 7.3$ & 2 & \\
\hline
\end{tabular}

Key: BMI, Body Mass Index; SD, Standard deviation.

Table 2. The average scores attained for the six mood subscales, the total mood disturbance score in professional driving sample $(n=120)$ and the normative values for an adult male sample [33] of the Profile of Mood States Questionnaire.

\begin{tabular}{lccc}
\hline Sub-scale & Total sample mean score & Normative values (Nyenhuis et al. [33]) & $p$ value \\
\hline Tension-anxiety & $9.5 \pm 6.5$ & $7.1 \pm 5.8$ & 0.003 \\
Anger-agression & $8.7 \pm 7.4$ & $7.1 \pm 7.3$ & 0.03 \\
Fatigue-inertia & $10.3 \pm 9.5$ & $7.3 \pm 5.7$ & $<0.001$ \\
Depression-dejection & $10.3 \pm 9.5$ & $7.5 \pm 9.2$ & 0.004 \\
Confusion-bewilderment & $7.0 \pm 4.9$ & $5.6 \pm 4.1$ & 0.062 \\
Vigor-activity & $14.4 \pm 5.9$ & $19.8 \pm 6.8$ & $<0.001$ \\
Total mood disturbance & $29.2 \pm 32.1$ & $14.8 \pm 32.7$ & $<0.001$ \\
\hline
\end{tabular}

Table 3. Becks Depression Inventory score for the total sample.

\begin{tabular}{ccc}
\hline & Total sample $(\mathrm{n}=120)$ & Cut-off for mild to moderate depression [25] \\
\hline BDI score & $9.7 \pm 7.9$ & 10 \\
\hline
\end{tabular}

Table 4. Mean HRV parameters for the professional driving sample during the baseline task.

\begin{tabular}{lc}
\hline HRV parameter & Total sample $(\mathrm{n}=120)$ \\
\hline $\mathrm{LF}\left(\mathrm{ms}^{2}\right)$ & $3.2 \pm 3.2$ \\
$\mathrm{HF}\left(\mathrm{ms}^{2}\right)$ & $2.9 \pm 3.3$ \\
Low total power $\left(\mathrm{ms}^{2}\right)$ & $3.7 \pm 3.8$ \\
LF:HF & $1.4 \pm 0.5$ \\
\hline
\end{tabular}

Key: HF, High frequency; HRV, Heart rate variability; LF, Low frequency; LF:HF, Low frequency to high frequency ratio (sympathovagal balance); $\mathrm{ms}^{2}$, Milliseconds squared.

Table 5. Correlations between Profile of Mood Sates scores, Beck's Depression Inventory and HRV parameters in cohort of professional drivers during a baseline task.

\begin{tabular}{lccc}
\hline & HRV parameter & $\mathrm{r}$ & $p$ \\
\hline \multirow{3}{*}{ Tension-anxiety } & LF & 0.25 & 0.046 \\
& HF & -0.24 & 0.044 \\
\multirow{2}{*}{ Anger-aggression } & Ratio & 0.24 & 0.049 \\
BDI & TP & 0.26 & 0.041 \\
& LF & 0.17 & 0.048 \\
\hline
\end{tabular}

Key: BDI, Becks Depression Inventory; HF, High frequency (normalised units); HRV, Heart rate variability; LF, Low frequency (normalised units); LF:HF, Low frequency to high frequency ratio (sympathovagal balance); TP, Total HRV power; $p$, Level of statistical significance; $r$, Correlation coefficient.
Table 6. Mean HRV parameters during the active task.

\begin{tabular}{lc}
\hline HRV parameter & Total sample $(\mathrm{n}=120)$ \\
\hline $\mathrm{LF}\left(\mathrm{ms}^{2}\right)$ & $3.4 \pm 3.5$ \\
$\mathrm{HF}\left(\mathrm{ms}^{2}\right)$ & $3.2 \pm 3.7$ \\
Low total power $\left(\mathrm{ms}^{2}\right)$ & $3.8 \pm 4.0$ \\
$\mathrm{LF}: \mathrm{HF}$ & $1.1 \pm 0.5$ \\
\hline Key: HF, High frequency; HRV, Heart rate variability; LF, \\
Low frequency; LF:HF, Low frequency to high frequency ra- \\
tio (sympathovagal balance); ms ${ }^{2}$, Milliseconds squared.
\end{tabular}

Table 7. Correlations between Profile of Mood Sates scores, Beck's Depression Inventory and HRV parameters in cohort of professional drivers during an active task.

\begin{tabular}{lccc}
\hline & HRV parameter & $\mathrm{r}$ & $p$ \\
\hline \multirow{2}{*}{ Confusion-bewilderment } & LF & 0.32 & 0.016 \\
& HF & -0.29 & 0.024 \\
\multirow{2}{*}{ Total mood disturbance } & LF & 0.24 & 0.049 \\
& Ratio & 0.27 & 0.038 \\
BDI & LF & 0.18 & 0.030 \\
& HF & -0.19 & 0.031
\end{tabular}

Key: BDI, Becks Depression Inventory; HF, High frequency (normalised units); HRV, Heart rate variability; LF, Low frequency (normalised units); LF:HF, Low frequency to high frequency ratio (sympathovagal balance); TP, Total HRV power; $p$, Level of statistical significance; $r$, Correlation coefficient. 
overseas. In this study, anxiety was found to be higher in the professional driving cohort than the advised normative values. This supports previous literature which suggests the inherent nature of professional driving lends itself to higher rates of anxiety [10-13]. In particular, perceived job stressors in the professional driving industry remain a primary source of anxiety [34], as does the burden of passenger safety [12], employment stability, and income [35]. Anxiety was also found to have a weak positive association with an increase in low frequency heart rate variability at baseline. Recent research suggests the LF bandwidth, while inclusive of sympathetic activity, may more accurately reflect a combination of autonomic (PSNS and SNS) system, and the baroreceptor reflex [36]. Nonetheless, increased LF has been associated with cardiac impairment, an increase risk of cardiac events and mortality [37]. The ratio of LF to HF, which has been suggested to reflect sympathovagal balance, was found to be positively correlated to anxiety scores. This indicates a parasympathetic nervous system withdrawal in the context of high resting anxiety levels. Further, HF HRV was found to be inversely related to anxiety symptoms. It is well accepted that HF is indicative of parasympathetic nervous system activity, and thus states of anxiety facilitate a parasympathetic withdrawal $[38,39]$. In times of acute stress, this provides an evolutionary benefit, allowing for an upregulation of sympathetic responses that enable an appropriate stress response. However, in the context of chronic stress, this sympathetic predominance is known to have deleterious effects on the heart. These include increases in circulating ionotropic and chronotropic catecholamines [40], suppression of vagal stimulation [41] and prolonged peripheral vasoconstriction, resulting in elevated resting total peripheral resistance, and thus elevated blood pressure [42]. These findings are novel within a population of Australian professional drivers and provide important insights into the potential associations between anxiety and cardiac health within this cohort. Steps to raise awareness, in particular in these males dominated industries, and the implementation of strategies to mitigate anxiety within this occupational group may improve not only the short-term psychological health of these individuals, but further, may improve their cardiovascular outcomes.

Depression scores were found to be associated with reductions in total heart rate variability, increased low frequency and reduced high frequency values. Given the similarities between anxiety and depression with regards to neurobiological aetiology and the continued scholarly support for the neurovisceral integration theory, it stands to reason that similar biological mechanism may be responsible. Reduced total heart rate variability has been well established as an independent predictor of not only cardiac health, but cardiovascular mortality [43]. Reduced total heart rate variability reflects a reduction in parasympathetic vagal outflow. The neurovisceral integration theory suggests efferent outputs from the pre-frontal cortex control vagal nerve inhibition of cardiac automaticity [44]. A reduction in this top-down control results in reduced cardiac control and is thus cardiopathogenic. The present study, in line with previous research, identified a bidirectional relationship between depression and reductions in total HRV at baseline. Given this, steps to address mental health conditions, in particular depression and anxiety, within the population of professional drivers would be beneficial to both psychological outcomes, as well as cardiovascular health. Further, given the hypothesised bidirectionality of this relationship, interventions that have been shown to improve total HRV, such as exercise, should be encouraged amongst this population.

There are some limitations of the present study. Response bias may be present due to the use of self-reported psychometric questionnaires; however, participants were advised that responses were deidentified to provide anonymity and reduce this bias While common in psychophysiological studies, univariate analysis has limitations in its predictive power, in particular as workplace variables rarely occur in isolation of one another. However, a rational and well recognised preliminary step is to investigate univariate associations, which allows the variables to be quantified preceding their insertion into a later predictive model. The primary objective is to include the "best" group of variables that increase predictive capability, whilst ensuring no unnecessary complexity. Further, although significant correlations were identified, the strength of these relationships should be examined in a larger cohort.

\section{Conclusions}

This study is the first to examine associations between mood states and cardiovascular functioning as measured by heart rate variability in Australian professional drivers. Anxiety and depression were both found to be associated with parasympathetic withdrawal, and sympathetic predominance, a known risk factor for CVD. This is of concern and should be considered when adopting holistic workplace health policies within this industry. Given the hypothesised bidirectionality of the relationship between mental health and cardiovascular function, it stands to reason that improvement of one state would symbiotically improve the other. That is, techniques to improve mental health states such as anxiety and depression may improve cardiovascular risk, and reciprocally, steps to improve cardiovascular variables, such as reducing LF HRV, may have beneficial effects on mental health. This study is important, as the psychophysiological health of Australia's professional drivers has been somewhat overlooked in the past. Given the large number of workers employed within this industry, and the potential personal and public safety implications of a suddenly unwell driver, it is vital that policies and workplace practices are designed to optimise the health of these individuals. 


\section{Author contributions}

Conceptualization, TC and SL; methodology, TC, SL; validation, TC, SL; formal analysis, TC, SL; investigation, TC; resources, TC, SL, data curation, TC, SL; writing-original draft preparation, TC; writing-review and editing, TC; supervision, SL.

\section{Ethics approval and consent to participate}

Ethics approval was granted by UTS HREC committee to conduct this study (No. 2014000110). Participants provide informed consent prior to inclusion in this study.

\section{Acknowledgment}

The authors acknowledge all participants who were included in this study. The authors also acknowledge Sydney Trains, Sydney Transport Union, Australia Post and the Transport Workers Union whom supported this research.

\section{Funding}

The author received a post-graduate research scholarship during her $\mathrm{PhD}$.

\section{Conflict of interest}

The authors declare no conflict of interest.

\section{References}

[1] Australian Institute of Health and Welfare. Mental health services in Australia: in brief 2018. Australian Government. 2018. Available at: https://www.aihw.gov.au/reports/mental-health-s ervices/mental-health-services-in-australia-in-brief-2018/repo rt-editions (Accessed: 3 August 2020).

[2] Australian Bureau of Statistics. 3218.0-Regional population growth, Australia, 2016. Canberra. 2017. Available at: https://www.abs.gov.au/AUSSTATS/abs@.nsf/Lookup/3218. 0Main+Features12016-17 (Accessed: 2 May 2020).

[3] Jeon HJ, Kim J, Kim B, Park SJ, Fava M, Mischoulon D, et al. Sleep quality, posttraumatic stress, depression, and human errors in train drivers: a population-based nationwide study in South Korea. Sleep. 2015; 37: 1969-1975.

[4] Farmer R, Tranah T, O'Donnell I, Catalan J. Railway suicide: the psychological effects on drivers. Psychological Medicine. 1992; 22: 407-414.

[5] Ro C, Shin T, Park M, Ku S. The relationship between depression, cognitive failure, mistakes, and accidents of the train drivers: the moderating effect of self-efficacy. Journal of the Korea Safety Management and Science. 2013; 15: 81-88.

[6] Ro C, Shin $\mathrm{T}$. The verification of causality among accident, depression, and cognitive failure of the train drivers. Journal of the Korea Society for Simulation. 2016; 25: 109-115.

[7] Zhou W, Gu G, Wu H, Yu S. Effects of occupational stress and related factors on depression symptoms in train drivers. Chinese Journal of Preventive Medicine. 2015; 49: 1080-1084.

[8] Hatami A, Vosoughi S, Hosseini AF, Ebrahimi H. Effect of codriver on job content and depression of truck drivers. Safety and Health at Work. 2020; 10: 75-79.

[9] Vakili M, Eslami Farsani S, Hossein S, Dehghani Tafti M. Prevalence of depression and its related factors among truck drivers in Yazd Province-2008. Iran Occupational Health. 2010; 6: 69-76.

[10] Hickey AR, Collins MD. Disinhibition and train driver performance. Safety Science. 2017; 95: 104-115.

[11] Mehnert A, Nanninga I, Fauth M, Schäfer I. Course and predictors of posttraumatic stress among male train drivers after the ex- perience of 'person under the train' incidents. Journal of Psychosomatic Research. 2012; 73: 191-196.

[12] Cothereau C. Professional and medical outcomes for French train drivers after "person under train" accidents: three year follow up study. Occupational and Environmental Medicine. 2004; 61: 488494.

[13] Boyce WS. Does truck driver health and wellness deserve more attention? Journal of Transport \& Health. 2016; 3: 124-128.

[14] Mansur ADP, Rocha MA, Leyton V, Takada JY, Avakian SD, Santos AJ, et al. Risk factors for cardiovascular disease, metabolic syndrome and sleepiness in truck drivers. Arquivos Brasileiros De Cardiologia. 2016; 105: 560-565.

[15] Ronna BB, Thiese MS, Ott U, Effiong A, Murtaugh M, Kapellusch $\mathrm{J}$, et al. The association between cardiovascular disease risk factors and motor vehicle crashes among professional truck drivers. Journal of Occupational \& Environmental Medicine. 2016; 58: 828832.

[16] Chapman J, Naweed A, Wilson C, Dorrian J. Sleep for heart health: investigating the relationship between work day sleep, days off sleep, and cardiovascular risk in Australian train drivers. Industrial Health. 2019; 57: 691-700.

[17] Australian Institute of Health and Welfare. Evidence for chronic disease risk factors. 2016. Available at: https://www.aihw.gov.a $\mathrm{u} /$ reports/chronic-disease/evidence-for-chronic-disease-risk-f actors/behavioural-and-biomedical-risk-factors (Accessed: 19 August 2020).

[18] Hare DL, Toukhsati SR, Johansson P, Jaarsma T. Depression and cardiovascular disease: a clinical review. European Heart Journal. 2014; 35: 1365-1372.

[19] Penninx BWJH. Depression and cardiovascular disease: epidemiological evidence on their linking mechanisms. Neuroscience \& Biobehavioral Reviews. 2017; 74: 277-286.

[20] Bucciarelli V, Caterino AL, Bianco F, Caputi CG, Salerni S, Sciomer S, et al. Depression and cardiovascular disease: the deep blue sea of women's heart. Trends in Cardiovascular Medicine. 2020; 30: 170-176.

[21] Zuzarte P, Duong A, Figueira ML, Costa-Vitali A, Scola G. Current therapeutic approaches for targeting inflammation in depression and cardiovascular disease. Current Drug Metabolism. 2018; 19: 674-687.

[22] Shao M, Lin X, Jiang D, Tian H, Xu Y, Wang L, et al. Depression and cardiovascular disease: Shared molecular mechanisms and clinical implications. Psychiatry Research. 2020; 285: 112802.

[23] Llinares R, Clifford GD. A spatio-temporal study of ischemia and the time-frequency coupling variations between the ST amplitude, heart rate and dominant angle. Institute of Electrical and Electronics Engineers. 2010.

[24] Craig A, Hancock K, Craig M. The lifestyle appraisal questionnaire: a comprehensive assessment of health and stress. Psychology \& Health. 1996; 11: 331-343.

[25] Beck AT, Steer RA, Brown G. Beck depression inventory-II. PsycTESTS Dataset. 2011.

[26] McNair DM. Profile of mood states T2. Educational and Industrial Testing Service. 1992. Available at: https://ci.nii.ac.jp/naid/ 20001520436/en/ (Accessed: 6 September 2020).

[27] Beck AT, Steer RA, Brown GK. BDI-II, Beck depression inventory: manual. 2nd edn. Boston: Harcourt, Brace, and Company. 1996.

[28] Griffith NM, Szaflarski JP, Szaflarski M, Kent GP, Schefft BK, Howe SR, et al. Measuring depressive symptoms among treatment-resistant seizure disorder patients: POMS Depression scale as an alternative to the BDI-II. Epilepsy \& Behavior. 2005; 7: 266-272.

[29] Thomas L, Krebs C. A review of statistical power analysis software. Bulletin of the Ecological Society of America. 1997; 78: 128 139.

[30] Cohen J. Statistical power analysis for the behavioral sciences. UK, London: Routledge. 2013.

[31] Lachin JM. Introduction to sample size determination and power 
analysis for clinical trials. Controlled Clinical Trials. 1981; 2: 93113.

[32] Heart Foundation of Australia. Your BMI 2010. Available at: http://www.heartfoundation.org.au/healthy-eating/pages/bm i-calculator.aspx (Accessed: 18 August 2020).

[33] Nyenhuis DL, Yamamoto C, Luchetta T, Terrien A, Parmentier A. Adult and geriatric normative data and validation of the profile of mood states. Journal of Clinical Psychology. 1999; 55: 79-86.

[34] Naweed A, Chapman J, Allan M, Trigg J. It comes with the job: work organizational, job design, and self-regulatory barriers to improving the health status of train drivers. Journal of Occupational and Environmental Medicine. 2018; 59: 264-273.

[35] Hege A, Lemke MK, Apostolopoulos Y, Whitaker B, Sönmez S. Work-life conflict among us long-haul truck drivers: Influences of work organization, perceived job stress, sleep, and organizational support. International Journal of Environmental Research And Public Health. 2019; 16: 984.

[36] Bar-Haim Y, Lamy D, Pergamin L, Bakermans-Kranenburg MJ, van IJzendoorn MH. Threat-related attentional bias in anxious and nonanxious individuals: a meta-analytic study. Psychological Bulletin. 2007; 133: 1-24.

[37] Yadav RL, Yadav PK, Yadav LK, Agrawal K, Sah SK, Islam MN. Association between obesity and heart rate variability indices: an intuition toward cardiac autonomic alteration-a risk of CVD. Diabetes, Metabolic Syndrome and Obesity: Targets and Therapy. 2019; 10: 57-64.

[38] Carney RM, Freedland KE, Stein PK. Anxiety, depression, and heart rate variability. Psychosomatic Medicine. 2000; 62: 84-87.

[39] Kemp AH, Quintana DS, Felmingham KL, Matthews S, Jelinek HF. Depression, comorbid anxiety disorders, and heart rate variability in physically healthy, unmedicated patients: implications for cardiovascular risk. PLoS ONE. 2012; 7: e30777.

[40] Goldstein DS. Catecholamines and stress. Endocrine Regulations. 2003; 37: 69-80.

[41] Porges SW. Cardiac vagal tone: a physiological index of stress. Neuroscience and Biobehavioral Reviews. 1995; 19: 225-233.

[42] Jern S. Effects of acute carbohydrate administration on central and peripheral hemodynamic responses to mental stress. Hypertension. 1992; 18: 790-797.

[43] Kemp AH, Quintana DS, Gray MA, Felmingham KL, Brown K, Gatt JM. Impact of depression and antidepressant treatment on heart rate variability: a review and meta-analysis. Biological Psychiatry. 2010; 67: 1067-1074.

[44] Thayer JF, Lane RD. A model of neurovisceral integration in emotion regulation and dysregulation. Journal of Affective Disorders. 2000; 61: 201-216. 